\title{
Ocorrências de quedas em idosos e a polifarmácia
}

\author{
Occurrences of falls in the elderly and polypharmacy \\ Ocurrencias de cándas en idosos y polifarmacia
}

Flávia Vilas Boas Ortiz Carli1*, Vinicius Dias dos Anjos ${ }^{1}$, Adriano Antônio da Silva ${ }^{1}$, Viviane Canhizares Evangelista ${ }^{1}$, Silvia Helena Soares Gianini ${ }^{1}$, Márcia Abúsio Cardin $^{1}$, Ligia Elaine Morelatto de Pieri da Silva1, Tereza Laís Menegucci Zutin¹.

\section{RESUMO}

Objetivo: Avaliar a ocorrência de queda em idosos e a presença da Polifarmácia. Métodos: Trata-se de um estudo transversal, realizado em um Centro Dia de idosos em uma cidade no interior do estado de São Paulo. Os seguintes critérios foram utilizados: os idosos independentes ou parcialmente dependentes, com idade de 60 anos ou mais, de ambos os sexos, com capacidade cognitiva preservada, foram considerados aptos a participarem da pesquisa, sendo que, os idosos com qualquer tipo de demência e acamados foram excluídos. A coleta de dados foi realizada por meio da aplicação de questionário de identificação de quedas e escala de Downton. Resultados: O estudo foi composto por 20 idosos, sendo $45 \%$ do sexo feminino. Cerca de $90 \%$ dos idosos mencionaram queda no ano anterior e $60 \%$ apresentavam a Polifarmácia. Conclusão: A queda tornou-se um grave problema de saúde pública, tal evento para ser prevenidos, exige profissionais qualificados para lidar com esta população, portanto, a identificação de preditores de quedas pode contribuir para a redução deste evento.

Palavras Chaves: Acidentes por quedas, Uso de medicamentos, Idoso.

\begin{abstract}
Objective: To evaluate the occurrence of falls in the elderly and the presence of polypharmacy. Methods: This is a cross-sectional study conducted in a center of the elderly in a city in the countryside of the state of São Paulo. The following criteria were used: The independent or partially dependent elderly, aged 60 years or older, of both sexes, with preserved cognitive capacity were considered able to participate in the research, and the elderly with any type of dementia and bedridden were excluded. Data collection was performed through the application of a fall identification questionnaire and a Downton scale. Results: The study consisted of 20 elderly individuals, $45 \%$ of whom were females. About $90 \%$ of the elderly reported falling in the previous year and $60 \%$ presented polypharmacy. Conclusion: The fall has become a serious public health problem, such an event to be prevented, requires qualified professionals to deal with this population, therefore, the identification of predictors of falls can contribute to the reduction of this event.
\end{abstract}

Key words: Accident by falls, Use of medications, Elderly.

\section{RESUMEN}

Objetivo: Evaluar la ocurrencia de caídas en los ancianos y la presencia de polifarmacia. Métodos: Este es un estudio transversal realizado en un centro de ancianos en una ciudad en el campo del estado de Sao Paulo. Se utilizaron los siguientes criterios: Los ancianos independientes o parcialmente dependientes, de 60

${ }^{1}$ Universidade de Marília (UNIMAR), Marília-SP. *E-mail: flaviavvilasboas@gmail.com 
años o más, de ambos sexos, con capacidad cognitiva preservada se consideraron capaces de participar en la investigación, y los ancianos con Se excluyó cualquier tipo de demencia y postrado en cama. La recopilación de datos se realizó mediante la aplicación de un cuestionario de identificación de caídas y una escala Downton. Resultados: El estudio consistió en 20 personas de edad avanzada, el $45 \%$ de las cuales eran mujeres. Alrededor del $90 \%$ de los ancianos reportaron caída en el año anterior y el $60 \%$ presentaron polifarmacia. Conclusión: La caída se ha convertido en un grave problema de salud pública, tal evento que debe prevenirse, requiere profesionales calificados para hacer frente a esta población, por lo tanto, la identificación de los predictores de caídas puede contribuir a la reducción de este evento.

Palabras clave: Accidente por caídas, Uso de medicamentos, Ancianos.

\section{INTRODUÇÃO}

Nos últimos anos, foi observado aumento considerável da expectativa de vida e, consequentemente, da população idosa (LIMA RJ, et al., 2017). No Brasil a Política Nacional do Idoso, define a pessoa idosa, como aquela que possui 60 anos ou mais (ALVES T, et al., 2017). Estima-se a existência de aproximadamente 17,6 milhões de pessoas idosas, e esta população crescerá 16 vezes até 2025, um aumento de mais de 33 milhões, classificação em sexto lugar no ranking mundial a respeito à população idosa (MIRANDA GM, et al., 2018).

No mundo, o envelhecimento é visto como um grande sucesso para a sociedade, contudo, um dos desafios é esquivar-se de empecilhos que impossibilitem o alcance do bem-estar e da qualidade de vida (ARAÚJO NETO AH, et al., 2017). O acelerado fenômeno nos países de baixa e média renda determina uma importante alteração na morbimortalidade pelo aumento da prevalência das doenças crônicas não transmissíveis (VIEIRA LS, et al., 2018). Em decorrência de tais transformações, surge a necessidade de se estudar melhor os problemas aos quais os idosos estão expostos, com destaque os eventos associados às quedas (PEREIRA SG, et al., 2017).

Pode-se compreender a queda como um deslocamento do corpo para uma posição inferior a inicial, sendo as causas deste evento multifatoriais. Em decorrência de sua alta incidência, complicações e custos elevados a queda é considerada um desafio para a saúde pública no Brasil (CHEHUEN NETO JÁ, et al., 2018). As quedas podem ocasionar perda de autonomia no idoso, podendo ter diferentes consequências, como lesões leves, fraturas e óbito. Tais consequências, aumentam o uso de recursos pessoais e materiais nos serviços de saúde, por esse motivo, a queda é considerada a lesão de maior custo entre os idosos (PEREIRA SG, et al., 2017). Cerca de 30 a $60 \%$ dos indivíduos acima dos 65 anos apresentam histórico de queda anualmente e, metade destes, relata mais de um evento, aumentando em até $70 \%$ a chance de cair novamente no próximo ano (ORCINO JL, et al., 2018).

As quedas são as principais causas de lesões relacionadas com visitas aos departamentos de emergência dos Estados Unidos e da etiologia primária de mortes acidentais em pessoas com idade superior a 65 anos. A taxa de mortalidade por quedas aumenta dramaticamente com a idade em ambos os sexos e em todos os grupos raciais e étnicos, representando $70 \%$ das mortes acidentais em pessoas de 75 anos de idade e mais velhos. Mais $90 \%$ das fraturas de quadril ocorreu em resultado de quedas, a maioria dessas em pessoas acima de setenta anos de idade (FULLER FG, 2002). A senescência e a senilidade são complexos. O indivíduo idoso apresenta peculiaridades na manifestação de seus sintomas. Doenças associadas, crônicas e irreversíveis, polifarmácia, alterações cognitivas, psicológicas, distúrbios de marcha e equilíbrio e fragilidade podem influir na manifestação do medo de cair em idosos. (MACEDO BG, et al., 2005).

A ocorrência de queda se associa a um conjunto de fatores extrínsecos e intrínsecos. Revisão de literatura sobre o tema aponta os principais fatores de risco: sexo feminino, maior idade, polifarmácia, uso de psicotrópicos, história prévia de queda, dificuldade visual, declínio cognitivo e fatores ambientais, como pisos escorregadios, mobílias e tapetes mal posicionados (PRATO C, et al., 2017). Podem ocorrer alterações na marcha, nos mecanismos de manutenção da postura e na força muscular que implicam em maior possibilidade de tropeços e, consequentemente, de quedas (ALVES T, et al., 2017). Estima-se que $23 \%$ da 
população brasileira consomem $60 \%$ da produção nacional de medicamentos, principalmente as pessoas acima de 60 anos (SILVA RD, 2012). Com o aumento da expectativa de vida da população, aumenta o contingente de portadores de doenças crônicas não transmissíveis (DCNT), que demandam assistência contínua e na qual os medicamentos têm um papel importante (CARVALHO MF, 2012).

A polifarmácia é a administração de cinco ou mais medicamentos a um paciente, fato esse vem aumentando nos últimos anos, mais especificamente na população idosa, em razão de estes pacientes serem alvo deste tipo de evento, decorrente das patologias múltiplas apresentadas. Este caso ganhou relevância nos Estados Unidos, quando a prática de tratar os idosos sem polifarmácia tornou-se um grande desafio para os clínicos. Por sua vez a etiologia da polifarmácia é multifatorial. Todavia, as alterações fisiológicas, a manifestação clínica e as doenças crônicas, principalmente as renais e hepáticas fazem com que os pacientes se tornem mais vulneráveis a sofrerem consequências das interações medicamentosas. $O$ objetivo do medicamento visa primordialmente gerar qualidade de vida e amenizar e controlar doenças. Porém, o uso descontrolado e excessivo pode levar o paciente a intoxicações e interações perigosas e prejudiciais à saúde (SECOLI SR, 2010).

Inúmeros estudos sobre a utilização de medicamentos, consideram que as quedas podem estar relacionadas a utilização de múltiplos fármacos. Neste contexto, segundo Brito FC et al. (2001), fica claro que a utilização de medicamentos como diuréticos, psicotrópicos, anti-hipertensivos e antiparkinsonianos, podem produzir efeitos colaterais nos idosos, que por sua vez, pode estar associado as causas inerentes de queda. O mais preocupante, contudo, é a falta de análise dos profissionais da saúde sobre os efeitos que esses medicamentos podem provocar nos idosos, como por exemplo, diminuição da função motora, fraqueza muscular, vertigem ou hipotensão postural. Em todo este processo, fica claro a importância da revisão contínua das queixas e dos receituários fornecidos pelo idoso em si, principalmente na Atenção Básica de Saúde

As implicações da influência dos medicamentos nas quedas muitas vezes não são reconhecidas pelo paciente, familiar, nem tampouco pelos profissionais, principalmente quando a polifarmácia é muito complexa. Deste modo, é indispensável que os profissionais conheçam os medicamentos potencialmente interativos, com o objetivo de prevenir os acidentes decorrentes da combinação terapêutica e ou potencialização desse uso (TOMAZ AS, et al., 2017).

Estudos comparativos apontam que a Fall Risk Score de Downton se destacou por possuir uma sensibilidade de $81,8 \%$, uma especificidade de $24,7 \%$, sendo que o tempo para ser realizado é de aproximadamente 6,45 minutos. Esta escala foi desenvolvida por Downton em 1992, e disponibilizada publicamente em seu livro Falls in the elderly em 1993. Segundo o autor, esta escala apresentou associação entre a pontuação obtida com o número de quedas. Portanto, esse instrumento pode ser utilizado para avaliar o risco de quedas em idosos. O instrumento é amplamente utilizado, tanto internacional como nacionalmente, devido fácil aplicação. Cinco critérios são utilizados na avaliação: quedas anteriores, medicamentos, déficit sensorial, estado mental e marcha. Pontuações iguais ou superiores a três indicam risco para queda, sendo que, a pontuação da escala varia de zero a onze (SCHIAVETO FV, 2008).

O estudo sobre queda no Brasil ainda é considerado escasso, visando à qualidade de vida que tem aumentado com os avanços da medicina e o aumento do consumo de medicamentos pela população de idosos. O presente estudo tem como objetivo analisar a ocorrência de queda em idosos e a presença da polifarmácia.

\section{MÉTODOS}

Trata-se de um estudo transversal, realizado em um Centro dia de idosos em uma cidade no interior do estado de São Paulo. A amostra foi selecionada a partir da análise de prontuários e foram utilizados os seguintes critérios: foram considerados aptos a participarem da pesquisa idosos independentes ou parcialmente dependentes, com idade de 60 anos ou mais, de ambos os sexos, com capacidade cognitiva preservada, sendo que, os critérios de exclusão foram idosos com qualquer tipo de demência e acamados. 
A coleta de dados foi realizada pelos acadêmicos do curso de enfermagem, por meio da aplicação do Questionário de identificação de quedas, o qual contém questões abertas e fechadas relacionadas a identificação pessoal, uso de medicações e a presença de quedas anteriores e suas características, tais informações foram coletadas junto aos idosos e consulta de prontuários.

A escala de Downton foi utilizada para avaliação do risco de quedas, que incluía questões como: uso de medicação (diuréticos, hipotensores, antiparkinsonianos, antidepressivos e hipertensores), alterações sensoriais (auditivas, visuais e nos membros), quedas anteriores e grau de orientação. Obtendo-se uma soma de três pontos ou mais, o idoso apresenta risco de quedas (BIAZUS M, 2010). A pesquisa em questão pode apresentar limitações em decorrência do método de questionamentos recordativos utilizado para a obtenção do histórico de queda no último ano, visto que os idosos podem ter apresentado um possível viés de memória. Para a realização da análise de dados e tabelas de frequências foi utilizado o programa estatístico SPSS.

Todos os princípio éticos da pesquisa com seres humanos foram seguidos, sendo este estudo aprovado pelo Comitê de Ética em Pesquisa da Universidade de Marília, em 25 de maio de 2017, sob o protocolo n: 2.083.533.

\section{RESULTADOS}

Foram incluídos 20 idosos no estudo, com relação às informações sociodemográficas, evidenciou-se que, a maioria da amostra foi composta por mulheres, viúvas, com idade entre 71 a 80 anos e que residem com os filhos (Tabela 1).

Tabela 1 - Dados sociodemográficos dos idosos que participaram do estudo. SP, 2018.

\begin{tabular}{|c|c|c|}
\hline Variáveis & $\mathbf{F}$ & $\%$ \\
\hline \multicolumn{3}{|l|}{ Sexo } \\
\hline Feminino & 15 & 75 \\
\hline Masculino & 5 & 25 \\
\hline \multicolumn{3}{|l|}{ Idade } \\
\hline $60-70$ & 3 & 15 \\
\hline $71-80$ & 9 & 45 \\
\hline $81-90$ & 4 & 20 \\
\hline $91-100$ & 4 & 20 \\
\hline \multicolumn{3}{|l|}{ Estado civil } \\
\hline Viúvo (a) & 16 & 80 \\
\hline Divorciado (a) & 2 & 10 \\
\hline Casado (a) & 1 & 5 \\
\hline Solteiro (a) & 1 & 5 \\
\hline \multicolumn{3}{|l|}{ Mora com } \\
\hline Filho (a) & 12 & 60 \\
\hline Sozinho (a) & 4 & 20 \\
\hline Neto (a) & 2 & 10 \\
\hline Esposo (a) & 1 & 5 \\
\hline Patroa & 1 & 5 \\
\hline
\end{tabular}

Fonte: Carli FVBO, et al., 2019. 
As quedas aconteceram em $90 \%$ dos idosos, totalizando 45 episódios em um ano e $70 \%$ referiram medo de cair novamente. Entre os idosos que sofreram quedas, evidenciou-se que $55 \%$ dos episódios ocorrem na rua, enquanto deambulavam e $55 \%$ tiveram que ser encaminhados ao pronto atendimento, pois $33 \%$ apresentavam ferimentos, tendo como consequência mais grave as fraturas (Tabela 2).

Tabela 2 - Características das quedas sofridas pelos idosos. SP, 2018.

\begin{tabular}{llr}
\hline Variáveis & $\mathbf{F}$ & $\%$ \\
\hline Local da queda & 7 & 39 \\
Dentro de casa & 10 & 55 \\
Na rua & 1 & 5 \\
Igreja & & \\
Encaminhamento & 10 & 55 \\
$\quad$ Pronto atendimento & 2 & 11 \\
Hospitalização & 6 & 33 \\
Tratamento local & & \\
Consequências & 6 & 33 \\
Ferimentos & 5 & 28 \\
Fraturas & 3 & 17 \\
Dor intensa & 4 & 22 \\
Sem consequências graves & 4 & \\
\hline
\end{tabular}

Fonte: Carli FVBO, et al., 2019.

$\mathrm{Na}$ Figura 1 encontram-se dispostos os medicamentos mais utilizados pelos idosos. Foram encontrados 100 tipos de fármacos com predomínio dos anti-hipertensivos, seguido de hipotensores, tranquilizantes, sedativos e antidepressivos.

Figura 1 - Medicamentos mais utilizados pelos idosos que participaram do estudo. SP, 2018.

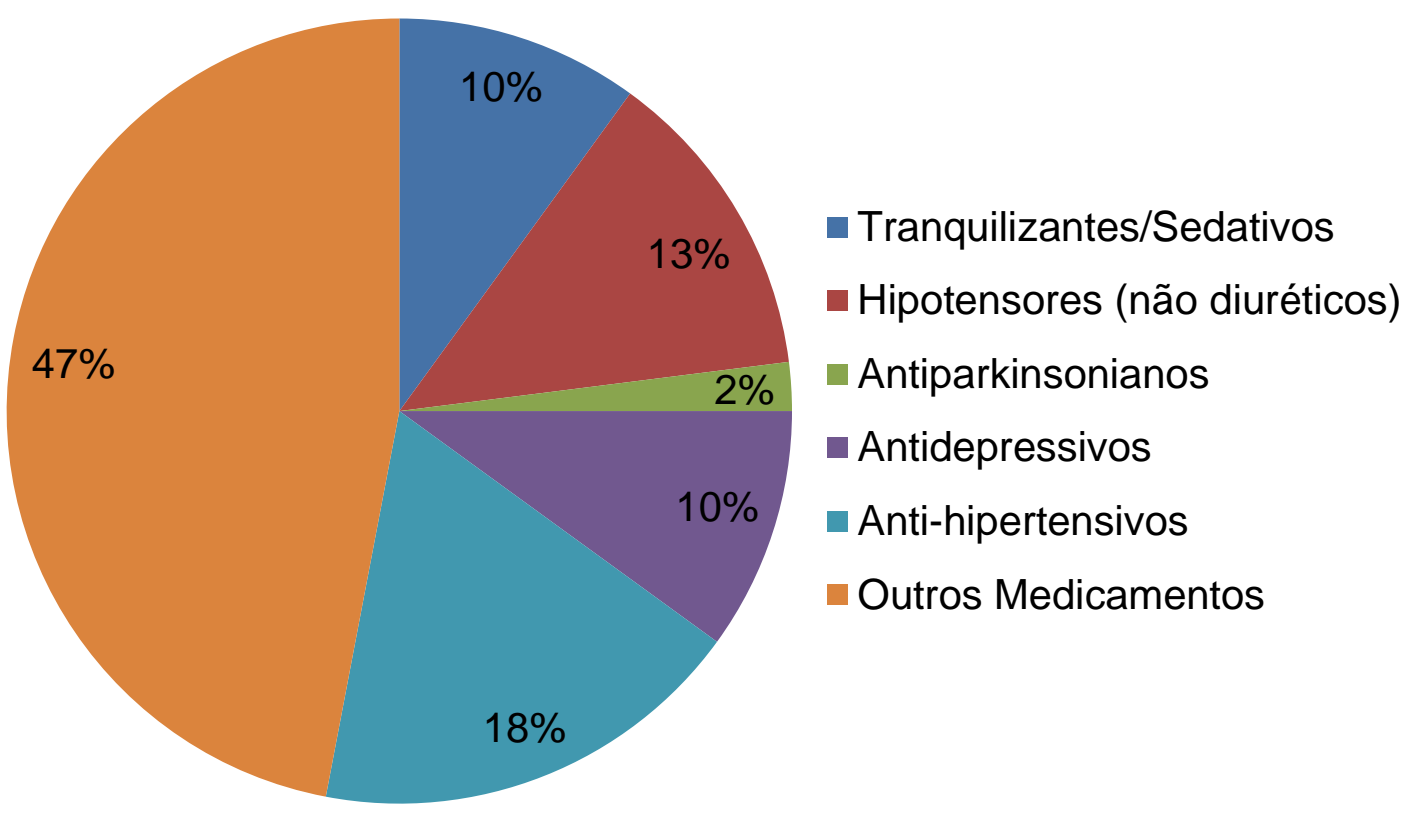

Fonte: Carli FVBO, et al., 2019. 
Em relação ao histórico de quedas, observamos na Tabela 3 que $90 \%$ dos idosos apresentaram queda no ano anterior e em 12 idosos a Polifarmácia estava presente.

Tabela 3 - Presença de queda e polifarmácia. SP, 2018.

\begin{tabular}{lll}
\hline Variáveis & Sim & \multicolumn{1}{c}{ Não } \\
\hline Polifarmácia & $12(60 \%)$ & $8(40 \%)$ \\
História previa de queda & $18(90 \%)$ & $2(10 \%)$
\end{tabular}

Fonte: Carli FVBO, et al., 2019.

\section{DISCUSSÂO}

Os dados encontrados referentes à queda em idosos revelou uma ocorrência bem elevada em relação a outros estudos semelhantes (VIEIRA LS, et al., 2018; REIS KM e JESUS CA, 2017; ARAÚJO NETO AH, et al., 2017). A pesquisa mostrou uma prevalência do sexo feminino, o que pode estar associado a maior expectativa de vida desse gênero, destacando que as mulheres possuem uma fragilidade musculoesquelética maior em comparação ao sexo masculino. Outros estudos que avaliaram queda em idosos também constataram uma prevalência do sexo feminino (HAMRA A, et al., 2007; SOUZA LH, 2017; MACHADO TR, 2009; FERREIRA LM, et al., 2019; AMORIM JS, et al., 2019). Dada a importância de se ressaltar que as mulheres possuem um envolvimento maior com as atividades domésticas e tarefas do dia-a-dia, como por exemplo, ir à feira/supermercado e a padaria (ANTES DL, et al., 2013).

Os fatores responsáveis por ocasionarem o evento queda, tem sido classificado na literatura, como intrínsecos, ou seja, fatores relacionados ao envelhecimento, concomitantemente as doenças e manifestações clínicas associados e também como fatores extrínsecos, que está intimamente ligado a barreiras físicas e ambientais e que limitam o decorrer das atividades exercidas pelos idosos. (FABRICIO SC, 2004).

Estudos apontam como um dos principais cenários de queda o próprio ambiente doméstico, ou seja, os idosos caem dentro de casa por causas multifatoriais (CRUZ D, et al., 2017; VIEIRA LS, et al., 2018). Por outro lado, o presente estudo identificou como cenário de maior ocorrência na rua, onde os idosos relataram queda durante a deambulação. Fato este comprovado por Oliveira AS et al. (2014) que demonstra em seu estudo que as ocorrências de queda têm sucedido em locais como: calçadas, meio-fio e ruas, jardins, varandas/sacadas, parques, garagem e estacionamento. Explica-se devido a boa capacidade funcional e a familiaridade que os idosos possuem com esses locais, que podem apresentar; superfícies molhadas/escorregadias, desníveis no chão e problemas com degraus e iluminação inadequada, tudo isso coopera para que o idoso seja envolvido em eventos como tropeço e escorregões.

Ferreira LM et al. (2019) relata que os eventos de queda devem ser não somente associados a fatores ambientais, mas também a fatores de saúde que o idoso apresenta, como por exemplo, a incontinência urinária, refletindo positivamente ao evento de queda; devido a quantidade de vezes que o mesmo levanta para ir ao banheiro, podendo neste trajeto tropeçar e cair, fato este aumenta consideravelmente no período da noite quando a baixa luminosidade e a acuidade visual ficam extremamente prejudicada. Rodrigues IG et al. (2014) aponta em seu estudo que a melhor maneira de se prevenir a queda nestes locais é promover a segurança através da utilização de piso não escorregadios, iluminação adequada, ausência de tapetes, barras de proteção, entre outros.

Nos últimos anos, pesquisas vêm buscando uma reflexão mais profunda sobre o ambiente acessível, o qual não só o idoso, mas todas as pessoas com alguma deficiência ou dificuldade de locomoção, possam usar plenamente, com segurança, conforto e eficiência. A presença de acessibilidade no meio urbano é uma exigência constitucional, cujo objetivo deve se permitir ganhos de autonomia e de mobilidade a uma porção maior de pessoas, incluindo aquelas que tenham dificuldades de locomoção, para que possam usufruir dos espaços urbanos com mais segurança, confiança e comodidade (PRADO AR, 2008). 
Freire RC et al. (2013) identificou em seu estudo que dentre as dificuldades encontradas pelos idosos em relação a locomoção, foi o trânsito e os problemas nas calçadas. Mais da metade das pessoas relataram que é difícil atravessar a rua, e que o principal problema é a impaciência dos motoristas. Em um estudo realizado no Rio Grande do Sul, com 466 idosos em Instituições de longa permanência constatou-se o encaminhamento para o hospital em $56 \%$ dos casos em que idosos sofrem acidentes por quedas, resultando em fraturas $19,2 \%$ (DUCA GF, 2013). Dados semelhantes ao nosso estudo, no qual $55 \%$ foram encaminhados para atendimento hospitalar, tendo como consequências mais graves as fraturas $28 \%$.

Como bem nos assegura Fabricio SC (2004), em sua pesquisa, os idosos que sofrem queda, geralmente em um mês acabam indo a óbito, devido a causas multifatoriais, taxa está que chega a $42,8 \%$. O mais preocupante, contudo, é constatar que a queda traz consigo inúmeras consequências, muitas das vezes complexas e difíceis de serem tratadas, como por exemplo, fratura de fêmur, pneumonia, lesão por pressão, entre outras. Neste contexto, é evidente que a qualidade de vida dos sobreviventes do episódio, irá reduzir drasticamente, podendo limitar até mesmo as habilidades em executar as Atividades de Vida Diária (AVD). Em todo este processo é de extrema importância a participação da equipe multidisciplinar na reabilitação deste idoso, aumentando assim, significativamente a taxa de sobrevida dos idosos afetados por queda.

Após a queda, o idoso pode desenvolver o sentimento de medo, referente a ocorrência de novos episódios, como também podem surgir o medo da hospitalização, da imobilidade e, principalmente, de tornarse dependente para a realização de AVD. Segundo Owings TM et al. (1999), é comum a imobilidade e invalidez após a hospitalização ocasionada por uma queda. A AVD pode apresentar limitações após a queda, o que acaba exigindo um maior suporte familiar. No presente estudo foi constatado que grande parte dos idosos que sofreram uma ou mais queda, desenvolveram o medo de cair novamente, o que contribui para o isolamento social e o comprometimento do sistema musculoesquelético, pois muitos idosos reduzem a mobilidade por medo de sofrerem novos episódios.

A queda é considera um evento real na vida da pessoa idosa e traz com ela inúmeras consequências (FABRICIO SC, 2004), que segundo Fhon JR et al. (2013), podem ser: lesões graves, incluindo fraturas, escoriações e ferimentos, fatos esses que podem gerar um alto custo para a sociedade e custo significativos para a família, em razão de modificação do ambiente para recebe-lo em casa. Antes DL et al. (2013), identificou que as consequências as quais mais prevaleceram, foram as escoriações/aranhão, circunstâncias está, não veio limitar os idosos em suas atividades de vida diária, mais trouxe consequências psicológicas e o medo de cair novamente; este sentimento suscita no idoso causas psicológicas e socias, tais como: incapacidade no caminhar, depressão, sentimentos de abandono e isolamento social. Maia BC et al. (2011), vem para certificar que os idosos acometidos pelo medo de cair novamente, traz consigo o receio de machucar-se, ser hospitalizado, sofrer imobilizações, ter declínio de saúde e ficar dependente de cuidados; em contra partida o medo pode atuar como medida protetora na vida do idoso, uma vez que ele passa a adotar comportamentos preventivos.

A Polifarmácia é considera como o uso de cinco ou mais medicamentos (SECOLI SR, 2010; SILVA RD, et al., 2012; CARVALHO MF, et al., 2012; ALMEIDA NA, et al., 2017). A pesquisa revelou que $60 \%$ dos idosos que sofreram queda a polifarmácia esteve presente. Estudo semelhante associa a polifarmácia e a queda em $69,7 \%$ dos idosos (REIS KM e JESUS CA, 2017). Fato este que pode ser explicado por interações medicamentosas que geram efeitos colaterais e levam os idosos a sofrerem queda. Rodrigues IG et al. (2014), explica que quanto maior o número de medicamentos o indivíduo utiliza, mais propenso a queda ele está, ressalta ainda que é preciso analisar os fármacos de forma individualizada, devido aos efeitos colaterais e interações medicamentosas que os mesmos propiciam.

Estudo realizado por Araújo Neto $\mathrm{AH}$ et al. (2017), com 45 idosos relacionou os medicamentos antihipertensivos ao risco de queda, pois podem ocasionar instabilidade postural, tornando-o como fator predisponente a este episódio. Quando comparado a presente pesquisa observamos que a utilização de medicamentos anti-hipertensivos atingiu $18 \%$ da totalidade para controle desta patologia. Semelhante ao presente estudo, o medicamento mais utilizado pelos idosos na pesquisa de Fabricio SC. (2004), foram os anti-hipertensivos. Por outro lado, Rodrigues IG et al. (2014), associa a classe dos psicoativos a queda, explica

REAS/EJCH | Vol.Sup.37 | e1082 | DOI: https://doi.org/10.25248/reas.e1082.2019 Página 7 de 9 
ainda que eles causam efeitos como: sedação, hipotensão postural, tremores, relaxamento muscular e fraqueza. Destaca ainda a classe dos diuréticos, associando-os aos distúrbios hidroeletrolíticos.

Bretam $O$ et al. (2013), traz que 30 a $50 \%$ dos idosos brasileiros possuem doenças metabólicas como hiperglicemia, dislipidemia e hipertensão arterial e essas comorbidades tem causado efeitos colaterais como a síncope (desmaio ou perda temporária e súbita de consciência), fato este que é fator predisponente a ocorrência de queda.

Estudo realizado utilizando-se a escala de Downton revelou que existe correlação entre a quantidade de medicamentos ministrados e o risco de queda, pois $33,33 \%$ dos pacientes utilizavam vários medicamentos e apresentava escore elevado na escala de Downton, o que representa grande risco às quedas (FERREIRA NC et., 2011). Neste contexto, fica evidente que o profissional médico, estabeleça uma rotina de reavaliação dos fármacos utilizados pelos idosos, visando assim uma pequena taxa de interação medicamentosa, ajustando a dosagem que o mesmo utiliza e diminuindo drasticamente a ocorrência de queda entre os pacientes desta classe (FABRICIO SC, 2004).

Várias publicações sugerem que sejam feitos estudos prospectivos para minimizar tal viés (LOURENÇO MA, 2013; MARQUES WV, 2014). A relevância deste estudo está na análise dos medicamentos prescritos, pois todos os prontuários dos pacientes foram analisados em relação à presença da polifarmácia nos idosos.

\section{CONCLUSÃO}

Pode-se concluir que a ocorrência de quedas em pacientes que apresentaram polifarmácia, ou seja, o uso 5 medicamentos foi elevada, com predomínio em idosas, com média de idade entre 71 a 80 anos, viúvas, sendo que a maioria sofreu quedas na rua enquanto deambulavam, tendo como principal consequência ferimentos e fraturas. O país está em processo de envelhecimento, essa tendência atual contribui para um número crescente de indivíduos idosas que, apesar de viverem mais, apresentam maiores condições crônicas o que leva ao tratamento com polifármacos, que quando associadas pode levar a um maior risco de queda. A queda tornou-se um grave problema de saúde pública, tal realidade exige profissionais que estejam preparados para lidar com as novas necessidades dessa crescente população, portanto a identificação de preditores de quedas pode contribuir para a redução desse evento.

\section{REFERÊNCIAS}

1. ALMEIDA NA, et al. Prevalência e fatores associadas à polifarmácia entre os idosos residentes na comunidade. Rev. Bras. Geriatr. Gerontol, 2017; 20(1): 143-153.

2. ALVES T, et al. Avaliação dos fatores de risco que contribuem para queda em idosos. Rev. Bras. Geriatr. Gerontol, 2017; 20(1): 59-69.

3. AMORIN JS, et al. Marcadores inflamatórios e ocorrência de quedas: coorte de idosos de Bambuí. Rev Saúde Pública, 2019; 53-35.

4. ANTES DL, et al. Circunstâncias e consequências das quedas em idosos de Florianópolios. EpiFlorida Idoso 2009. Rev Bras Epidemiol, 2013; 16(2): 469-81.

5. ARAÚJO NETO AH et al. Quedas em idosos institucionalizados: riscos, consequências e antecedentes. Rev Bras Enferm, 2017; 70(4): 752-8.

6. BIAZUS M, et al. Avaliação do Risco de Quedas em Idosos. RBCEH, 2010; 7(1): 34 - 41.

7. BRETAN O, et al. Risco de queda em idosos da comunidade: avaliação com o teste Timed up and go. Braz J Otorhinolaryngol, 2013; 79(1): 18-21.

8. BRITO FC, COSTA SM. Quedas. In: Papaleo Netto M, Brito FC. Urgências em geriatria. São Paulo: Ed. Atheneu, 2001; 323-35.

9. FERREIRA NC, et al. Correlação entre mobilidade funcional, equilíbrio e risco de quedas em idosos com doença de Parkinson. Geriatria \& Gerontologia, 2011; 5(2): 74-9.

10. CARVALHO MF, et al. Polifarmácia entre idosos do município de São Paulo - Estudo SABE. Rev Bras Epidemiol, 2012; 15(4); 817-27.

11. CHEHUEN NETO JÁ, et al. Percepção sobre queda e exposição de idosos a fatores de risco domiciliares. Ciência \& Saúde Coletiva, 2018 23(4): 1097-1104. 
12. CRUZ D, et al. Fatores associados a quedas recorrentes em uma coorte de idosos. Cad. Saúde Colet, $2017 ; 25$ (4): 475-482.

13. DUCA GF, et al. Quedas e fraturas entre residentes de instituições de longa permanência para idosos. Rev Bras Epidemiol, 2013; 16(1): 68-76.

14. FABRíCIO SC. Causas e consequências de quedas de idosos atendidos em hospital público. Rev Saúde Pública, 2004; 38(1): 93-99.

15. FERREIRA LM, et al. Quedas recorrentes e fatores de risco em idosos institucionalizados. Ciência \& Saúde Coletiva, 2019; 24(1): 67-75.

16. FHON JR, et al. Prevalência de quedas de idosos em situação de fragilidade. Ver Saúde Pública, 2013; 47(2): 26673.

17. FREIRE RC, et al. Estudo da acessibilidade de idosos ao centro da cidade de Caratinga, MG. Rev. Bras. Geriatr. Gerontol, 2013; 16(3): 541-558.

18. FULLER FG. Falls in the elderly. American Family Physician, 2002; 61(1): 2159-2174.

19. HAMRA A, et al. Correlação entre fratura por queda em idosos e uso prévio de medicamentos. ACTA ORTOP BRAS, 2007; 15(3); 143-145.

20. LIMA RJ, et al. Capacidade funcional e o risco de quedas em pessoas idosas. Rev Rene. 2017; 18(5): 616-22.

21. LOURENÇO MA. Análise da ocorrência e do risco de quedas por meio de testes físicos em pacientes com artrite reumatoide. [dissertação]. Marília (SP): Faculdade de Medicina de Marília. 2013. 56 p.

22. MACEDO BG, et al. Clinical parameters to detec the fear of falling down, inside the elderly. Physical Therapy in Movement, 2005; 18(3)18: 5-70.

23. MACHADO TR, et al. Avaliação da presença de risco para queda em idosos. Rev. Eletr. Enf, 2009; 11(1): 32-8.

24. MAIA BC, et al. Consequências das Quedas em Idosos Vivendo na Comunidade. Ver. Bras. Geriatr. Gerontol., Rio de Janeiro, 2011; 14(2): 381-393.

25. MARQUES WV, et al. Influência da capacidade funcional no risco de quedas em adultos com artrite reumatoide. Rev Bras Reumatol, 2014; 54(5): 404-8.

26. MIRANDA GM, et al. O envelhecimento populacional brasileiro: desafios e consequências sociais atuais e futuras. Rev. Bras. Geriatr. Gerontol, 2016; 19(3): 507-519.

27. OLIVEIRA AS, et al. Fatores ambientais e risco de quedas em idosos: revisão sistemática. Rev. Bras. Geriatr. Gerontol, Rio de Janeiro, 2014; 17(3): 637-645.

28. ORCINO JL, et al. Avaliação do risco de quedas em mulheres saudáveis entre 40 e 90 anos. IV Congresso de Ensino. 2018.

29. OWINGS TM, et al. Exercise: Is it a solution to falls by older adults? Journal of Applied Biomechanics, 1999;15(1):5663.

30. PEREIRA SG, et al. Prevalência de quedas no domicílio de longevos e fatores extrínsecos associados. Rev. LatinoAm. Enfermagem, 2017; 25.

31. PRADO AR, coordenadora. Município acessível ao cidadão. São Paulo: Fundação Prefeito Faria Lima - CEPAM; 2001. 32. PRATO C, et al. Frequência e fatores associados a quedas em adultos com 55 anos e mais. Rev Saúde Pública, 2017; $51(37): 11$.

33. REIS KM, JESUS CA. Relação da polifarmácia e polipatologia com a queda de idosos instituicionalizados. Texto Contexto Enferm, 2017; 26(2).

34. RODRIGUES IG, et al. Quedas em idosos: fatores associados em estudo de base populacional. Rev Bras Epidemol, Jul-Set, 2014: 705-718.

35. SCHIAVETO FV. Avaliação do risco de quedas em idosos na comunidade. [Dissertação]. Escola de Enfermagem de Ribeirão Preto da Universidade de São Paulo. 2008. 117f.

36. SECOLI SR. Polifarmácia: interações e reações adversas no uso de medicamentos por idosos. Revista Brasileira de Enfermagem, 2010; 63(1): 36-140.

37. SILVA RD, et al. Polifarmácia em geriatria. Revista de AMRIGS, 2012 ; 52 (2): 164-174.

38. SOUZA LH, et al. Queda em idosos e fatores de risco associados. Rev. Aten. Saúde, 2017; v. 15, n. 54, p. 55-60.

39. TOMAZ SA, et al. Prevalência de quedas em idosos devido ao uso de benzodiazepínicos e diuréticos. Revista UNINGÁ, 2017;52(1): 34-39.

40. VIEIRA LS, et al. Quedas em idosos no Sul do Brasil: prevalências e determinantes. Rev Saúde Publica, 2018; 52:22. 